UPR-672-T

\title{
Dyonic BPS Saturated Black Holes of Heterotic String on a Six-Torus
}

\author{
Mirjam Cvetič ${ }^{*}$ and Donam Youm ${ }^{\dagger}$ \\ Physics Department \\ University of Pennsylvania, Philadelphia PA 19104-6396
}

(July 1995)

\begin{abstract}
Within effective heterotic superstring theory compactified on a six-torus we derive minimum energy (supersymmetric), static, spherically symmetric solutions, which are manifestly invariant under the target space $O(6,22)$ and the strong-weak coupling $S L(2)$ duality symmetries with 28 electric and 28 magnetic charges subject to one constraint. The class of solutions with a constant axion corresponds to dyonic configurations subject to two charge constraints, with purely electric [or purely magnetic] and dyonic configurations preserving $\frac{1}{2}$ and $\frac{1}{4}$ of $N=4$ supersymmetry, respectively. General dyonic configurations in this class have a space-time of extreme Reissner-Nordström black holes while configurations with more constrained charges have a null or a naked singularity.
\end{abstract}

04.50.+h,04.20.Jb,04.70.Bw,11.25.Mj

*E-mail address: cvetic@cvetic.hep.upenn.edu

${ }^{\dagger}$ E-mail address: youm@cvetic.hep.upenn.edu 
There has been an accumulating evidence for the strong-weak coupling duality (referred to as the $S$ duality) in string theory (See for example Refs. [1,2]), which relates supersymmetric vacua of a strongly coupled theory to supersymmetric vacua of a dual - weakly coupled - theory. Deeper understanding of these duality symmetries would provide us with a handle on the non-perturbative nature of superstring theory.

In four-dimensions, string vacua with $N=4$ low energy supersymmetry are conjectured to be self-dual, i.e., the string vacua of the heterotic string compactified on a six-torus transform into each other under the $S L(2, Z)$ transformations 1 . The $S L(2, Z)$ symmetry acts on charges, the axion and the four-dimensional dilaton field, whose value determines the string loop expansion parameter and parameterizes the strength of the string coupling. In addition, the string world-sheet action can be cast in a manifestly $O(6,22)$ symmetric form [5.,6], referred to as the target space $T$ duality symmetry.

Evidence for the $S$ duality conjecture of $N=4$ supersymmetric string vacua has been provided by demonstrating the $S$ duality invariance of quantities which are believed not to be modified by string quantum corrections, e.g., the low energy effective field theory [7,6], allowed spectrum of electric and magnetic charges, Yukawa couplings between massless scalars and massive charged states as well as the Bogomol'nyi-Prasad-Sommerfeld (BPS) saturated mass spectrum of the corresponding non-trivial configurations in the effective theory [8,9].

The BPS saturated states within the effective theory compactified on a six-torus have been addressed for states with special charge configurations (See for example Refs. [8, 10, [13]), which in turn prevented one from establishing the full symmetry structure of such configurations as well as the full nature of their singularity structure. In this paper, we shall present the explicit form of general BPS saturated (supersymmetric), spherically symmetric, static configurations in the effective heterotic string theory compactified on a six-torus at generic points of moduli space, which can be obtained from the generating solution with, among scalar fields, only diagonal internal metric and the dilaton turned on. The BPS saturated spectrum is both $O(6,22)$ and $S L(2, \mathbb{R})$ invariant 2 . In addition, the explicit form of these configurations allows for a synthetic analysis of their singularity structures and their thermal properties within the class of solutions.

The effective field theory of massless bosonic fields for heterotic string on a Narain torus 114 can be obtained by compactifying the ten-dimensional $N=1$ supergravity theory coupled to $N=1$ super-Maxwell theory on a six-torus [6.15]. The ten-dimensional bosonic fields are given by $\hat{G}_{M N}, \hat{B}_{M N}, \hat{A}_{M}^{I}$ and $\Phi(0 \leq M, N \leq 9,1 \leq I \leq 16)$, which correspond to ten-dimensional metric, two-form field, gauge fields of $U(1)^{16}$, and the dilaton field, respectively. The field strengths of $\hat{A}_{M}^{I}$ and $\hat{B}_{M N}$ are defined as $\hat{F}_{M N}^{I}=\partial_{M} \hat{A}_{N}^{I}-\partial_{N} \hat{A}_{M}^{I}$ and $\hat{H}_{M N P}=\partial_{M} \hat{B}_{N P}-\frac{1}{2} \hat{A}_{M}^{I} \hat{F}_{N P}^{I}+$ cyc. perms., respectively.

\footnotetext{
${ }^{1}$ The heterotic string compactified on a six-torus is conjectured to be dual to the Type IIA string compactified on a $T^{2} \times K_{3}$ surface; this duality has its origin in the string-string duality conjecture [1] [4] of the heterotic and the Type IIA string theory in six-dimensions.

${ }^{2}$ At the quantum level, these symmetries are integer valued.
} 
The Kaluza-Klein compactification [16] of the original ten-dimensional action on a sixtorus is obtained with the following Ansatz for the Zehnbein: $\hat{E}_{M}^{A}=\left(\begin{array}{cc}e^{\frac{\phi}{2}} e_{\mu}^{\alpha} & A_{\mu}^{(1) m} e_{m}^{a} \\ 0 & e_{m}^{a}\end{array}\right)$, where $A_{\mu}^{(1) m}(m=1, \ldots, 6)$ are Kaluza-Klein $U(1)$ gauge fields and $\phi \equiv \Phi-\ln \operatorname{det} e_{m}^{a}$ is the four-dimensional dilaton field.

The four-dimensional action [6,15] for massless bosonic fields contains the following fields: the graviton $g_{\mu \nu}$, the dilaton $\phi, 28 U(1)$ gauge fields $\mathcal{A}_{\mu}^{i} \equiv\left(A_{\mu}^{(1) m}, A_{\mu m}^{(2)}, A_{\mu}^{(3) I}\right)$ defined as $A_{\mu m}^{(2)} \equiv \hat{B}_{\mu m}+\hat{B}_{m n} A_{\mu}^{(1) n}+\frac{1}{2} a_{m}^{I} A_{\mu}^{(3) I}, A_{\mu}^{(3) I} \equiv \hat{A}_{\mu}^{I}-a_{m}^{I} A_{\mu}^{(1) m}$ with the field strengths $\mathcal{F}_{\mu \nu}^{i}=\partial_{\mu} \mathcal{A}_{\nu}^{i}-\partial_{\nu} \mathcal{A}_{\mu}^{i}$, the two form field ${ }^{3} B_{\mu \nu}$ with the field strength given by $H_{\mu \nu \rho}=$ $e_{\mu}^{\alpha} e_{\nu}^{\beta} e_{\rho}^{\gamma} \hat{E}_{\alpha}^{M} \hat{E}_{\beta}^{N} E_{\gamma}^{P} \hat{H}_{M N P}=\partial_{\mu} B_{\nu \rho}-\frac{1}{2} \mathcal{A}_{\mu}^{i} L_{i j} \mathcal{F}_{\nu \rho}^{j}+$ cyc.perms., and a symmetric $O(6,22)$ matrix $M$ of scalar fields, which can be expressed in terms of the following $O(6,22)$ matrix

$$
V=\left(\begin{array}{c}
V^{I} \\
V^{I I} \\
V^{I I I}
\end{array}\right)=\left(\begin{array}{ccc}
E^{-1} & -E^{-1} C & -E^{-1} a^{T} \\
0 & E & 0 \\
0 & a & I_{16}
\end{array}\right)
$$

as $M=V^{T} V$, where $E \equiv\left[e_{m}^{a}\right], C \equiv\left[\frac{1}{2} \hat{A}_{m}^{I} \hat{A}_{n}^{I}+\hat{B}_{m n}\right]$ and $a \equiv\left[\hat{A}_{m}^{I}\right] . \quad V$ plays a role of a Vielbein in the $O(6,22)$ target space.

The 4 -d effective action is invariant under the $O(6,22)$ transformations [6, 15]:

$$
M \rightarrow \Omega M \Omega^{T}, \quad \mathcal{A}_{\mu}^{i} \rightarrow \Omega_{i j} \mathcal{A}_{\mu}^{j}, \quad g_{\mu \nu} \rightarrow g_{\mu \nu}, \quad \phi \rightarrow \phi .
$$

Here, $\Omega \in O(6,22)$, i.e., $\Omega^{T} L \Omega=L$, where $L$ is an $O(6,22)$ invariant matrix. In addition, the corresponding equations of motion and Bianchi identities have the invariance under the $S L(2, \mathbb{R})$ transformations [17, 15]:

$$
S \rightarrow S^{\prime}=\frac{a S+b}{c S+d}, M \rightarrow M, g_{\mu \nu} \rightarrow g_{\mu \nu}, \mathcal{F}_{\mu \nu}^{i} \rightarrow \mathcal{F}_{\mu \nu}^{\prime i}=(c \Psi+d) \mathcal{F}_{\mu \nu}^{i}+c e^{-\phi}(M L)_{i j} \tilde{\mathcal{F}}_{\mu \nu}^{j}
$$

where $S \equiv \Psi+i e^{-\phi}, \tilde{\mathcal{F}}^{i \mu \nu}=\frac{1}{2}(\sqrt{-g})^{-1} \varepsilon^{\mu \nu \rho \sigma} \mathcal{F}_{\rho \sigma}^{i}$ and $a, b, c, d \in \mathbb{R}$ satisfy $a d-b c=1$.

The ten-dimensional supersymmetry transformations for gravitino $\psi_{M}$, dilatino $\lambda$ and 16 gaugini $\chi^{I}$ are expressed in terms of the four-dimensional fields as:

$$
\begin{aligned}
\delta \hat{\psi}_{\mu} & =\partial_{\mu} \varepsilon+\frac{1}{4} \omega_{\mu \beta \gamma} \gamma^{\beta \gamma} \varepsilon+\frac{1}{4} e_{\mu}^{\alpha} \eta_{\alpha[\beta} e_{\gamma]}^{\nu} \partial_{\nu} \phi \gamma^{\beta \gamma} \varepsilon-\frac{1}{8} e^{-\phi} H_{\mu \nu \rho} \gamma^{\nu \rho} \varepsilon \\
& +\frac{1}{8}\left(e_{b}^{n} \partial_{\mu} e_{n c}-e_{c}^{n} \partial_{\mu} e_{n b}\right) I \otimes \Gamma^{b c} \varepsilon-\frac{1}{4} e^{-\frac{\phi}{2}}\left[(V L)_{c i}^{I}+(V L)_{c i}^{I I}\right] \mathcal{F}_{\mu \nu}^{i} \gamma^{\nu 5} \otimes \Gamma^{c} \varepsilon, \\
\delta \psi_{d} & =-\frac{1}{4} e^{-\frac{\phi}{2}}\left[e_{d}^{m} \partial_{\mu} e_{m b}+e_{b}^{m} \partial_{\mu} e_{m d}-e_{d}^{m} e_{b}^{n}\left(\partial_{\mu} B_{m n}+\frac{1}{2} a_{m}^{I} \partial_{\mu} a_{n}^{I}-\frac{1}{2} a_{n}^{I} \partial_{\mu} a_{m}^{I}\right)\right] \gamma^{\mu 5} \otimes \Gamma^{b} \varepsilon \\
& -\frac{1}{8} e^{-\phi}\left[(V L)_{d i}^{I}-(V L)_{d i}^{I I}\right] \mathcal{F}_{\mu \nu}^{i} \gamma^{\mu \nu} \varepsilon
\end{aligned}
$$

\footnotetext{
${ }^{3}$ The four-dimensional two-form field is equivalent to a pseudo-scalar (the axion) $\Psi$ through the duality transformation $H^{\mu \nu \rho}=-\frac{e^{2 \phi}}{\sqrt{-g}} \varepsilon^{\mu \nu \rho \sigma} \partial_{\sigma} \Psi$.
} 


$$
\begin{aligned}
\delta \lambda & =e^{-\frac{\phi}{2}} \partial_{\mu} \Phi \gamma^{\mu} \varepsilon-\frac{1}{6} e^{-\frac{3}{2} \phi} H_{\mu \nu \rho} \gamma^{\mu \nu \rho} \varepsilon-\frac{1}{2} e^{-\frac{\phi}{2}}\left(\partial_{\mu} B_{m n}+\frac{1}{2} a_{m}^{I} \partial_{\mu} a_{n}^{I}-\frac{1}{2} a_{n}^{I} \partial_{\mu} a_{m}^{I}\right) \gamma^{\mu} \otimes \Gamma^{m n} \varepsilon \\
& -\frac{1}{2} e^{-\phi}(V L)_{d i}^{I} \mathcal{F}_{\mu \nu}^{i} \gamma^{\mu \nu 5} \otimes \Gamma^{d} \varepsilon, \\
\delta \chi^{I} & =2 e^{-\frac{\phi}{2}} \partial_{\mu} a_{m}^{I} \gamma^{\mu 5} \otimes \Gamma^{m} \varepsilon+e^{-\phi}(V L)_{I i}^{I I I} \mathcal{F}_{\mu \nu}^{i} \gamma^{\mu \nu} \varepsilon,
\end{aligned}
$$

where $\delta \hat{\psi}_{\mu} \equiv \delta \psi_{\mu}-A_{\mu}^{(1) m} \delta \psi_{m}$ and $\delta \psi_{d} \equiv e_{d}^{m} \delta \psi_{m}$. Here, $\gamma^{\alpha}$ and $\Gamma^{a}$ satisfy the $O(1,3)$ and $O(6)$ Clifford algebras, respectively. The gamma matrices with curved indices are defined as $\gamma^{\mu} \equiv e_{\alpha}^{\mu} \gamma^{\alpha}$ and $\Gamma^{m} \equiv e_{a}^{m} \Gamma^{a}$.

Static configurations, which saturate the Bogomol'nyi bound for their masses, i.e., the minimum energy configurations in their class, correspond to bosonic backgrounds which preserve the supersymmetry transformations (4) [B], thus also referred to as supersymmetric configurations. The Killing spinor equations, which are obtained by setting the supersymmetry transformations (4) to zero, provide constraints on the Killing spinors $\varepsilon$ and a set of coupled first order differential equations for the supersymmetric bosonic backgrounds.

Our aim is to obtain general supersymmetric, spherically symmetric, static configurations with a general allowed charge content associated with the $28 U(1)$ gauge fields. The fourdimensional space-time metric is chosen to be of the form:

$$
g_{\mu \nu} d x^{\mu} d x^{\nu}=\lambda d t^{2}-\lambda^{-1} d r^{2}-R\left(d \theta^{2}+\sin ^{2} \theta d \phi^{2}\right)
$$

and the scalar fields $M, \phi$ and $\Psi$ depend on the radial coordinate $r$, only. The Maxwell's equations and Bianchi identities determine the $U(1)$ field strengths to be

$$
\mathcal{F}_{t r}^{i}=\frac{e^{\phi}}{R}\left[M_{i j} \tilde{Q}_{j}+\Psi(M L)_{i j} P_{j}\right], \quad \mathcal{F}_{\theta \phi}^{i}=P_{i} \sin \theta
$$

where $P_{i}$ 's correspond to the physical magnetic charges and the physical electric charges 18 are given by $Q_{i}=e^{\phi_{\infty}}\left[M_{i j \infty} \tilde{Q}_{j}+\Psi_{\infty}(M L)_{i j \infty} P_{j}\right]$.

One can show that with the above static, spherically symmetric Ansatz the Killing spinors are invariant under the $O(6,22)$ transformations and transform as $\varepsilon \rightarrow[\cos (\Delta / 2)+$ $\left.i \gamma^{5} \sin (\Delta / 2)\right] \varepsilon$ under the $S L(2, \mathbb{R})$. Here, $\tan \Delta=-c e^{-\phi} /(c \Psi+d)$. The first order differential equations are thus invariant under both transformations and therefore one can generate new class of supersymmetric solutions by imposing $O(6,22)$ and $S L(2, \mathbb{R})$ transformations on the known supersymmetric solution. One can bring the arbitrary asymptotic values of the scalar fields to the forms $M_{\infty}=I$ and $S_{\infty}=i$ by imposing the following $O(6,22)$ and $S L(2, \mathbb{R})$ transformations:

$$
M_{\infty} \rightarrow \hat{M}_{\infty}=\hat{\Omega} M_{\infty} \hat{\Omega}^{T}=I, \quad S_{\infty} \rightarrow \breve{S}_{\infty}=\left(a S_{\infty}+b\right) / d=i
$$

where $\hat{\Omega} \in O(6,22)$, ad $=1$, and in the quantized theory the charge lattice vectors live in the new transformed lattice. Then, the subsets of $O(6,22)$ and $S L(2, \mathbb{R})$ transformations that preserve the above new asymptotic values of $M$ and $S$ are $O(6) \times O(22)$ and $S O(2)$, respectively. To obtain solutions with arbitrary asymptotic values of $M$ and $S$, one has to undo the above transformations.

We are going to find the general solution for configurations where, from the scalar fields, only the diagonal internal metric and the dilaton field are non-zero. We shall refer to 
such configurations as generating ones, since all the other configurations in this class can be obtained by performing a subset of $O(6) \times O(22) \subset O(6,22)$ and $S O(2) \subset S L(2, \mathbb{R})$ transformations on the generating ones. Note, that configurations obtained in that manner have the same four-dimensional space-time structure and thus the same singularity and thermal properties as the generating solution.

The Killing spinor equations for the configuration with only non-zero scalar fields given by the diagonal internal metric $\left(e_{a}^{m}=\delta_{a}^{m} e_{a}\right)$ and the dilaton take the form:

$$
\begin{aligned}
\sqrt{\lambda} R\left[\partial_{r} \ln \lambda+\partial_{r} \phi\right] \varepsilon_{u, \ell} & = \pm \Sigma_{a=1}^{6}\left(\mathbf{Q}_{a}^{(1)}+\mathbf{Q}_{a}^{(2)}\right) \Gamma^{a} \varepsilon_{\ell, u}, \\
\sqrt{\lambda} R\left[\partial_{r} \ln \lambda-\partial_{r} \phi\right] \varepsilon_{u, \ell} & =i \Sigma_{a=1}^{6}\left(\mathbf{P}_{a}^{(1)}+\mathbf{P}_{a}^{(2)}\right) \Gamma^{a} \varepsilon_{\ell, u}, \\
2 \sqrt{\lambda} R \partial_{r} \ln e_{a} \Gamma^{a} \varepsilon_{u, \ell} & =\left[\mp\left(\mathbf{Q}_{a}^{(1)}-\mathbf{Q}_{a}^{(2)}\right)+i\left(\mathbf{P}_{a}^{(1)}-\mathbf{P}_{a}^{(2)}\right)\right] \varepsilon_{\ell, u}, \quad a=1, \cdots, 6, \\
\partial_{r} \sqrt{\lambda R} & =0,
\end{aligned}
$$

where $\mathbf{Q}_{a}^{(1)} \equiv e^{\frac{\phi}{2}} e_{a}^{m} \tilde{Q}_{m}, \mathbf{Q}_{a}^{(2)} \equiv e^{\frac{\phi}{2}} e_{m}^{a} \tilde{Q}_{6+m}, \mathbf{P}_{a}^{(1)} \equiv e^{-\frac{\phi}{2}} e_{m}^{a} P_{m}$, and $\mathbf{P}_{a}^{(2)} \equiv e^{-\frac{\phi}{2}} e_{a}^{m} P_{6+m}$. And from $\delta \chi^{I}=0$, one has $P_{I}^{(3)}=0=Q_{I}^{(3)}$. It can be shown 19 that out of $2 \cdot 28$ dyonic charges, only two magnetic and two electric charges can be non-zero with electric and magnetic charges arising from different $U(1)$ factors, with one set of electric and magnetic charges arising from the Kaluza-Klein sector and the other set arising from the two-form gauge fields with the same corresponding indices. Without loss of generality, we choose the non-zero charges to be $P_{1}^{(1)}, P_{1}^{(2)}, Q_{2}^{(1)}, Q_{2}^{(2)}$.

The upper $\varepsilon_{u}$ and lower $\varepsilon_{\ell}$ two-component spinors are subject to the constraints: $\Gamma^{1} \varepsilon_{u, \ell}=$ $i \eta_{P} \varepsilon_{\ell, u}$ if $\mathbf{P}_{1}^{(1)} \neq 0$ and/or $\mathbf{P}_{1}^{(2)} \neq 0$, and $\Gamma^{2} \varepsilon_{u, \ell}=\mp \eta_{Q} \varepsilon_{\ell, u}$ if $\mathbf{Q}_{2}^{(1)} \neq 0$ and/or $\mathbf{Q}_{2}^{(2)} \neq 0$. Here, $\eta_{P}$ and $\eta_{Q}$ are \pm 1 . Note, that non-zero magnetic and electric charges each break $\frac{1}{2}$ of the remaining supersymmetries. Thus, purely electric [or magnetic] configurations preserve $\frac{1}{2}$, while dyonic solutions preserve $\frac{1}{4}$ of $N=4$ supersymmetry in 4 dimensions. The first and the second sets of configurations fall into vector- and hyper-supermultiplets, respectively.

The explicit form for the static, spherically symmetric generating solution is given by 1 :

$$
\begin{aligned}
\lambda & =r^{2} /\left[\left(r-\eta_{P} P_{1}^{(1)}\right)\left(r-\eta_{P} P_{1}^{(2)}\right)\left(r-\eta_{Q} Q_{2}^{(1)}\right)\left(r-\eta_{Q} Q_{2}^{(2)}\right)\right]^{\frac{1}{2}}, \\
R & =\left[\left(r-\eta_{P} P_{1}^{(1)}\right)\left(r-\eta_{P} P_{1}^{(2)}\right)\left(r-\eta_{Q} Q_{2}^{(1)}\right)\left(r-\eta_{Q} Q_{2}^{(2)}\right)\right]^{\frac{1}{2}}, \\
e^{\phi} & =\left[\frac{\left(r-\eta_{P} P_{1}^{(1)}\right)\left(r-\eta_{P} P_{1}^{(2)}\right)}{\left(r-\eta_{Q} Q_{2}^{(1)}\right)\left(r-\eta_{Q} Q_{2}^{(2)}\right)}\right]^{\frac{1}{2}}, \\
g_{11} & =\left(\frac{r-\eta_{P} P_{1}^{(2)}}{r-\eta_{P} P_{1}^{(1)}}\right), g_{22}=\left(\frac{r-\eta_{Q} Q_{2}^{(1)}}{r-\eta_{Q} Q_{2}^{(2)}}\right), g_{m m}=1 \quad(m \neq 1,2) .
\end{aligned}
$$

Here, the radial coordinate is chosen so that the horizon is at $r=0$. The requirement that the ADM mass of the above configuration saturates the Bogomol'nyi bound restricts the choice of parameters $\eta_{P, Q}$ such that $\eta_{P} \operatorname{sign}\left(P_{1}^{(1)}+P_{1}^{(2)}\right)=-1$ and $\eta_{Q} \operatorname{sign}\left(Q_{2}^{(1)}+Q_{2}^{(2)}\right)=-1$,

\footnotetext{
${ }^{4}$ Such a solution is obtained along the similar lines as the generating solution for the supersymmetric, spherically symmetric solutions in Abelian Kaluza-Klein theory [19].
} 
thus yielding non-negative BPS saturated ADM mass of the form $M_{B P S}=\left|P_{1}^{(1)}+P_{1}^{(2)}\right|+$ $\left|Q_{2}^{(1)}+Q_{2}^{(2)}\right|$. In order to have regular $\mathrm{BH}$ solution with singularity behind or on the horizon, one has to choose the relative signs of two magnetic and two electric charges to be the same Ð. Thus, the solution has always nonzero BPS saturated ADM mass.

A general class of solutions with zero axion can be obtained from the generating ones by performing a subset of $O(6) \times O(22) \subset O(6,22)$ transformations which generate new types of solutions from the generating one. These transformations correspond to $S O(6) / S O(4)$ transformations with $\frac{6 \cdot 5-4 \cdot 3}{2}=9$ parameters and $S O(22) / S O(20)$ transformations with $\frac{22 \cdot 21-20 \cdot 19}{2}=41$ parameters, which along with the original 4 charges provide a configuration with $56-2=54$ charges; namely, those are configurations with 28 electric $\vec{Q}$ and 28 magnetic $\vec{P}$ charges subject to the following two constraints (in the basis where the asymptotic value of $M$ takes an arbitrary value):

$$
\vec{P}^{T} \mathcal{M}_{ \pm} \vec{Q}=0 \quad\left(\mathcal{M}_{ \pm} \equiv(L M L)_{\infty} \pm L\right)
$$

The $S O(2) \subset S L(2, \mathbb{R})$ transformation provides one with one more parameter $\tan \Delta=$ $-c e^{-\phi} /(c \Psi+d)$, which replaces the two constraints (10) with one $S L(2, \mathbb{R})$ and $O(6,22)$ invariant constraint on charges :

$$
\vec{P}^{T} \mathcal{M}_{-} \vec{Q}\left[\vec{Q}^{T} \mathcal{M}_{+} \vec{Q}-\vec{P}^{T} \mathcal{M}_{+} \vec{P}\right]-(+\leftrightarrow-)=0 .
$$

Therefore, the general configurations in this class have $2 \cdot 28-1=55$ charge degrees of freedom 9 .

The ADM mass for a general configuration in this class can be obtained from the one for the generating solutions and can be cast in the following $O(6,22)$ and $S L(2, \mathbb{R})$ invariant form 1]:

$$
M_{B P S}^{2}=e^{-\phi_{\infty}}\left\{\vec{P}^{T} \mathcal{M}_{+} \vec{P}+\vec{Q}^{T} \mathcal{M}_{+} \vec{Q}+2\left[\left(\vec{P}^{T} \mathcal{M}_{+} \vec{P}\right)\left(\vec{Q}^{T} \mathcal{M}_{+} \vec{Q}\right)-\left(\vec{P}^{T} \mathcal{M}_{+} \vec{Q}\right)^{2}\right]^{\frac{1}{2}}\right\} .
$$

\footnotetext{
${ }^{5}$ Note, that the case of opposite relative signs for the two electric charges [and two magnetic charges] and the equal magnitude of the two electric [and two magnetic charges] would yield zero ADM mass as pointed out in a related context by Hull and Townsend [20]. Such purely electrically (or purely magnetically) charged configurations were found and studied in Refs. [21], while dyonic ones and their implications for enhanced symmetries [20] at special points of moduli space were addressed in Ref. [22]. Such configurations are not regular; they have a naked singularity.

${ }^{6}$ The constraint (11) on charges signals that the obtained class of configurations may not be the most general supersymmetric one. This constraint is removed for the supersymmetric [non-extreme] states by applying an additional subset of $S O(8,24)$ transformations 13,23 on the corresponding supersymmetric [non-extreme] generating solutions. $O(8,24)$ is the symmetry of the effective threedimensional action for the corresponding stationary solutions. Analogous procedure was used [24] to generate all the black holes in Abelian Kaluza-Klein theory.

${ }^{7}$ We thank A. Sen for pointing out to us the procedure to derive such a mass.
} 
Note, that when the magnetic $\vec{P}$ and electric $\vec{Q}$ charges are parallel in the $S O(6,22)$ sense, this ADM mass is the bound for configurations that preserve $\frac{1}{2}$ of $N=4$ supersymmetry [8, $11,13,25,26$, i.e., the corresponding generating solution is either purely electric or purely magnetic. In the case when the magnetic and electric charges are not parallel, the mass is larger and the configurations preserve $\frac{1}{4}$ of $N=4$ supersymmetry.

We now turn to the discussion of the thermal and global space-time properties of such configurations, which can be classified according to the number of non-zero charges of the generating solutions:

- The case with all the four charges non-zero 9 corresponds to BH's with a horizon at $r=0$ and a time-like singularity hidden behind the horizon, i.e., the global spacetime structure is that of the extreme Reissner-Nordström BH's. The corresponding Hawking temperature $T_{H}=\left.\partial_{r} \lambda\right|_{r=0} /(2 \pi)$ is zero and the entropy ( $\equiv \frac{1}{4}$ of the area of the event horizon) is finite $S=\pi \sqrt{\left|P_{1}^{(1)} P_{1}^{(2)} Q_{2}^{(1)} Q_{2}^{(2)}\right|}$.

- The case with three nonzero charges corresponds to solutions with a singularity located at the horizon $(r=0), T_{H}=0$ and $S=0$.

- The case with two nonzero charges 9, say, $P_{1}^{(1)} \neq 0 \neq P_{1}^{(2)}$, corresponds to singular solutions with the horizon and the singularity coinciding at $r=0, T_{H}=1 /\left(4 \pi \sqrt{\left|P_{1}^{(1)} P_{1}^{(2)}\right|}\right)$ and $S=0$.

- The case with one nonzero charge 10 corresponds to BH's with a naked singularity, $T_{H}=\infty$ and $S=0$.

\section{ACKNOWLEDGMENTS}

The work is supported by U.S. DOE Grant No. DOE-EY-76-02-3071, the NATO collaborative research grant CGR 940870 and the National Science Foundation Career Advancement Award PHY95-12732. M.C. would like to thank M. Duff, G. Gibbons, J. Harvey, C. Hull,

\footnotetext{
${ }^{8}$ Solutions with two electric [and two magnetic] charges equal correspond to configurations with constant $M$. A class of such configurations was obtained by Kallosh et al. 10. For the case where all the four charges are equal, all the scalars are constant and the four-dimensional metric reduces to that of Reissner-Nordström BH's, which is also pointed out in Ref. [27].

${ }^{9}$ Configurations with two non-zero electric charges were constructed by Sen [13], and shown by Peet [26] to be supersymmetric. The supersymmetric configurations with $P_{1}^{(1)} \neq 0$ and $Q_{2}^{(1)} \neq 0$, found by the authors [19], correspond to configurations in the Kaluza-Klein sector of the theory.

${ }^{10} P_{1}^{(1)} \neq 0$ case corresponds to the Kaluza-Klein monopole solution of Gross and Perry, and Sorkin [28], and were shown to be supersymmetric by Gibbons and Perry [25]. The case when $P_{1}^{(2)} \neq 0$ corresponds to the $H$-monopole solution [12].
} 
D. Lüst and especially A. Sen for useful discussions and the Aspen Center for Theoretical Physics for hospitality during the completion of the work. 


\section{REFERENCES}

[1] See E. Witten, Nucl. Phys. B443 (1995) 85 and references therein.

[2] C. Hull and P. Townsend, Nucl. Phys. B438 (1995) 109.

[3] M.J. Duff, Nucl. Phys. B442 (1995) 47.

[4] M.J. Duff, S. Ferrara and R.R. Khuri, Phys. Lett. B356 (1995) 479; M.J. Duff, J.T. Liu and R. Minasian, Nucl. Phys. B452 (1995) 261; A. Sen, Nucl. Phys. B450 (1995) 103; J.A. Harvey and A. Strominger, Nucl. Phys. B449 (1995) 535.

[5] A. Tseytlin, Phys. Lett. B242 (1990) 163; Nucl. Phys. B350 (1991) 395.

[6] J. Maharana and J.H. Schwarz, Nucl. Phys. B390 (1993) 3.

[7] M. De Roo, Nucl. Phys. B255 (1985) 515.

[8] J. Harvey and J. Liu, Phys. Lett. B268 (1991) 40.

[9] A. Sen, Mod. Phys. Lett. A8 (1993) 2023; J. Schwarz and A. Sen, Phys. Lett. B312 (1993) 105.

[10] R. Kallosh, A. Linde, T. Ortin, A. Peet and A. Van Proeyen, Phys. Rev. D46 (1992) 5278; R. Kallosh and T. Ortin, Phys. Rev. D48 (1993) 742; T. Ortin, Phys. Rev. D47 (1993) 3136.

[11] T. Banks, M. Dine, H. Dijkstra and W. Fischler, Phys. Lett. B212 (1988) 45.

[12] R. Khuri, Phys. Lett. B259 (1991) 261; B294 (1992) 325; B387 (1992) 315; J. Gauntlett, J. Harvey and J. Liu, Nucl. Phys. B409 (1993) 363.

[13] A. Sen, Nucl. Phys. B440 (1995) 421; A. Sen, Mod. Phys. Lett. A10 (1995) 2081.

[14] K. Narain, Phys. Lett. B169 (1986) 41; K. Narain, H. Sarmadi and E. Witten, Nucl. Phys. B279 (1987) 369.

[15] A. Sen, Int. J. Mod. Phys. A9 (1994) 3707; A. Giveon, M. Porrati and E. Rabinovici, Phys. Rep. 244 (1994) 77.

[16] T. Kaluza, Sitz. Preuss. Akad. Wiss. Kl. (1921) 966; O. Klein, Z. Phys. 37 (1926) 895; Y.M. Cho and P.G.O. Freund, Phys. Rev. D12 (1975) 1711; J. Scherk and J.H. Schwarz, Nucl. Phys. B153 (1979) 61; M.J. Duff, NI-94-015 preprint (1994), hep-th \# 9410046, Talk given at The Oskar Klein Centenary Symposium, Stockholm, Sweden, 19-21 Sept. 1994.

[17] J.H. Schwarz, CALT-68-1815 preprint, hep-th \# 9209125; A. Sen, Nucl. Phys. B404 (1993) 109; A. Shapere, S. Trivedi and F. Wilczek, Mod. Phys. Lett. A6 (1991) 2677.

[18] E. Witten, Phys. Lett. B86 (1979) 283.

[19] M. Cvetič and D. Youm, Nucl. Phys. B438 (1995) 182, and addendum ibidem B449 (1995) 146.

[20] C.M. Hull and P.K. Townsend, Nucl. Phys. B451 (1995) 525.

[21] K. Behrndt, HUB-EP-95/6 preprint, hep-th \# 9506106; R. Kallosh, SU-ITP-95-12 preprint, hep-th \# 9506113; R. Kallosh and A. Linde, SU-ITP-95-14 preprint, hep-th \# 9507022.

[22] M. Cvetič and D. Youm, hep-th \# 9507160, Phys. Lett. B359 (1995) 87.

[23] A. Sen, Nucl. Phys. B434 (1995) 179.

[24] M. Cvetič and D. Youm, UPR-649-T preprint, hep-th \# 9503082, to be published in Phys. Rev. Lett.

[25] G. Gibbons and M. Perry, Nucl. Phys. B248 (1984) 629.

[26] A. Peet, PUPT-1548 preprint, hep-th \# 9506200, to be published in Nucl. Phys. B. 
[27] M.J. Duff and J. Rahmfeld, Phys. Lett. B345 (1995) 441.

[28] D.J. Gross and M.J. Perry, Nucl. Phys. B226 (1983) 29; R.D. Sorkin, Phys. Rev. Lett. 51 (1983) 87. 